\title{
Diverse Tree Mulberry Geometry and Cultural Practices Adopted by the Farmer's and its Optimization
}

\section{Megharaja, Harishkumar J, Chaithra KC, Likhithgowda M and Manjunatha HB*}

Department of Studies in Sericulture Science, University of Mysore, Mysuru, Karnataka, India

*Corresponding Author: Manjunatha HB, Department of Studies in Sericulture Science, University of Mysore, Mysuru, Karnataka, India.

Email: manjunathahb@gmail.com
Received: May 03, 2021

Published: May 26, 2021

(C) All rights are reserved by Manjunatha HB., et al.

\begin{abstract}
Currently, due to climate change, the paucity of water, and labor, farmers are navigating from bush system of mulberry cultivation to tree plantation regardless of standard and scientific recommendations. Keeping this transit in view, a systematic field visit based study was performed with personal interaction to explore knowledge level, skill, and scientific awareness of the farmers who were undertaking tree mulberry plantation and cultivation practices. Unexpectedly, while different spacing method ranging from $8^{\prime} \times 3^{\prime}$ to $10^{\prime}$ x $14^{\prime}$ ' has been followed by the farmers, about $62.86 \%$ of farmers were adopted $10^{\prime}$ x $10^{\prime}$ spacing for tree plantation. Height of the stump and branches, and a number of branches per plant in the first year and ensuing years were also varying among farmers, which explicit remarkable diversity in it. Additionally, the number of disease free layings of silkworm brushed as against quantum of leaves available in the tree mulberry garden also differs. Notably, as opined by the farmers, the quality and yield of cocoons produced using the leaves of tree plantation were comparatively better than the mulberry leaves harvested from the bush type of plantation despite diverse tree mulberry geometry and cultivation practices are in vogue. Considering these limitations, we have established a tree mulberry plantation with optimized plant geometry and evaluated its suitability. However, a systematic research is still offered to evolve appropriate technology for the long term and large scale tree mulberry cultivation with assured quality and sustained leaf and cocoon production.
\end{abstract}

Keywords: Mulberry; Cocoon; Seri-farmers; Tree Plantation; Water Scarcity

\section{Introduction}

An important component of the sericulture industry for the production of silk is mulberry foliage that serves as an exclusive source of nutrients for the silkworms (Bombyx mori L.) to biosynthesize proteinaceous fiber and spin in the form of cocoon. Mulberry (Morus spp.) being fast-growing deciduous perennial woody plant possesses deep rooting nature and grows under varied climatic conditions - temperate to tropical. Presently, mulberry has been cultivating in heterogeneous agro-climatic conditions following diverse cultivation methods and practices as a low bush with a comparatively closer spacing of $2^{\prime} \times 6^{\prime}$ to $3^{\prime} \times 3^{\prime}$ in plains of Southern and Western parts of India. In hilly areas of Jammu and Kashmir, depending on soil topography, it has been cultivating as a large/medium tree with the spacing of $5^{\prime} \times 5^{\prime}$ to $10^{\prime} \times 10^{\prime}$ [1]. However, when the mulberry plant is allowed to grow tall with a crown height of 5 to 6 from the ground level and stem girth of 4 to 5 inches referred to tree mulberry [2]. These plants are pruned once in a year during monsoon (July-August) at a height of 4' to 6' from the ground level and allowed to grow with a maximum of 8 to 10 shoots at the crown [3].
In recent years, the concept of tree mulberry cultivation is diffusing into plain areas as it is comparatively advantageous over bush plantation for sustainable foliage production and hassle-free cultural operations. This phenomenal change is due to the fact that a steep rise in annual mean temperature, irregular rainfall, declined groundwater resources, and scarcity of manpower. But, the bottleneck crisis is that no scientific basis of plantation method, cultural operations and precise package of practices are available for plain areas unlike hilly areas for tree plantation [4]. Therefore, Seri-farmers have been adopting varied plantation and cultivation practices as per their own knowledge, convenience and experience gained over the years. This unscientific approach not only affecting the accurate assessment of mulberry leaf production in a year or ensuing years but also to estimate the number of disease free layings of silkworm (pure breed/hybrid) required for rearing accordingly. Thus, it has created a big vacuum due to lack of substantial scientific innovations and necessitates a systematic evaluation for ground reality data on the tree mulberry plantation and practices that are being followed by the farmers. 
Keeping this gap and importance of tree mulberry plantation in view, a systematic study was undertaken not only to assess the knowledge level of the farmers on tree mulberry plantation, cultural operations, package of practices that are influencing cocoon production but also the problems encountered by them. Such that appropriate technology and plan of action shall be evolved for a long term and large scale cultivation of tree mulberry plantation with assured quality and sustained leaf production in plain areas of developing countries like India.

\section{Methodology}

\section{Field study areas}

The present study was carried out at well known traditional sericulture areas - Kolar and Chikkaballapura districts, which are located in the eastern region of Karnataka state, India. For this study, we have selected five taluks from Kolar i.e., Kolar, Srinivasapura, Malur, Bangarpet, and Mulbagal, and a taluk from Chikkaballapura district i.e., Chintamani, wherein most of the farmers are practicing tree mulberry plantation and silkworm rearing. In total 34 respondents were selected randomly and data was collected based on the set questioner and personal interview. All the data were analyzed using an appropriate statistical tool.

\section{New tree mulberry garden}

Considering the diversity prevailed in the tree mulberry garden, we have newly established a tree mulberry garden following the plant geometry of 8' x 5', stump height of 2.5' and pruning height from the ground level 3 to $3.5^{\prime}$. The mulberry variety used in the present study was $\mathrm{G}_{4}$, the dry leaves/twigs for mulching were collected on campus and green manure crop was sun hemp.

\section{Results}

Status of the farmers practicing tree mulberry cultivation

In the present study, for comparative analysis, Seri-farmers are categorized into three different groups based on the area of tree mulberry plantation they posses. Those who are having $<2.5$ acres of tree mulberry plantation are treated as small-scale farmers, between 2.5 to 5 acres as medium-scale farmers and $>5$ acres as large-scale farmers. Accordingly, $71.43 \%$ of total respondents were small farmers, $22.86 \%$ were medium farmers and $5.71 \%$ were large farmers (Figure 1A). All most all respondents practicing tree mulberry cultivation using bore wells and farm ponds as a source of irrigation although the survey areas fell under rain fed area (Figure 1B)

\section{Mulberry variety}

The mulberry varieties used for tree plantation were $V_{1}$ and $G_{4}$. Of which, $97.14 \%$ of the respondents were using $V_{1}$ variety while only $2.86 \%$ of farmers using $\mathrm{G}_{4}$ mulberry variety (Figure $1 \mathrm{C}$ ). More- over, all the farmers were using saplings as a source of materials for tree plantation (Figure 1D).

\section{Geometry of tree mulberry plantation}

Notably, the geometry of mulberry plantation plays a pertinent role in determining leaf quality, yield, and inter-cultural operations. Surprisingly, the present study revealed different spacing systems ranging from $8 \times 3$ ' to $10 \times 14^{\prime}$ being followed by the farmers in their tree mulberry gardens. Among them, a majority of $62.86 \%$ of farmers were adopted $10 \times 10^{\prime}$ spacing. While $8.57 \%$ of farmers followed $10 \times 6$ ' spacing, yet another group of $8.57 \%$ of farmers adopted $10 \times 8^{\prime}$ spacing for tree mulberry plantation. But, different spacing of $8 \times 3^{\prime}, 8 \times 8^{\prime}, 10 \times 3^{\prime}, 10 \times 6^{\prime}, 12 \times 10^{\prime}$ and $10 \times$ $14^{\prime}$ for tree plantation were followed by rest of the farmers (Figure $1 \mathrm{~F})$. It indicates undoubtedly the existence of vast diversity in the tree mulberry plantation, which has been adopted as per their own knowledge and convenience - opined by the farmers based on the present survey (Figure 1 and 2).

\section{Stump height in the tree plantation}

Surprisingly, the height of the stump maintained by the farmers in the tree mulberry garden was also inconsistent that ranges from $1.5^{\prime}$ to $4.0^{\prime}$ representing low and high (Figure 1G). Among, a large number of farmers accounting $31.42 \%$ maintain a medium stump height of 3', while 28.57 and $25.71 \%$ of farmers maintain the stump height of $3.5^{\prime}$ and $2.5^{\prime}$ in the tree mulberry plantation respectively. A low stump height of $1.5^{\prime}$ was maintained by $5.71 \%$ farmers, while the other $8.57 \%$ of farmers maintain a high stump height of $4^{\prime}$ in their mulberry tree plantation (Figure $1 \mathrm{G}$ ). The exact cause for this diversity albeit unclear, but as per the opinion of the farmers, it is based on their own convenience.

\section{Pruning height of the tree plantation}

As spacing, stump height, and pruning height are interlinked and optimization of all these cultural operations has to be achieved for maximization of foliage quality and yield. Surprisingly, in the present survey, such appropriate rationale among these cultural procedures was not noticed. As anticipated, Seri-farmers have been performing inconsistent levels of pruning at the field that resulted in the height of the tree mulberry varies from $2.5^{\prime}$ to $5.5^{\prime}$ from ground level. Among them, $25.71 \%$ of farmers practice pruning at a height of 4 , while others perform pruning at the height of 3.5' (20\%), 4.5' (20\%), 5' (17.14\%) and 5.5' (8.571\%). Besides, less number of farmers were undertaking pruning at the height of $2.5^{\prime}$ $(2.85 \%)$ and 3 ' (5.71\%) for tree mulberry (Figure $1 \mathrm{H}$ ) indicating the wide diversity.

\section{Number of shoots/tree maintained in the plantation}

Interestingly, while different spacing, stump, and pruning height in the tree mulberry garden was recorded in the present survey, 
the same inconsistency even in the number of shoots per plant was obvious that ranges from 30 to 120 shoots per tree/plant. For comparative analysis, we have classified arbitrarily the total number of shoots maintained in a tree by the farmers at the field as 0 to 30,30 to 60 , and 60 to 120 . Accordingly, 4, 5, and 6 branches per tree were found to be maintained by $70.59,11.76$, and $17.64 \%$ of farmers during the first year of tree mulberry plantation. Subsequently, number of shoots maintained in a mulberry tree was found increased as the plant grows. Eventually, about 30 shoots per tree were found to maintain by $44.11 \%$ farmers, whereas $50 \%$ of farmers maintained 30 to 60 shoots per plant and $11.62 \%$ of farmers retained 60 to 90 shoots per mulberry tree during the second year. Amazingly, while $40 \%$ of the farmers were maintaining 30 to 60 shoots per plant, 36.66 farmers retained 60 to 90 shoots per tree and $23.33 \%$ of farmers were found maintained the highest number 90 to 120 shoots per tree during the third year and onwards (Figure 2). The diversity in the number of shoots per tree, as per the present survey report, does not follow any scientific rule and the legitimacy in terms of biomass in the tree mulberry in relation to available soil nutrients and health is enigmatic.

Manures and fertilizers applied for tree mulberry plantation

As different spacing, stump and pruning height, and a number of shoots per mulberry tree were recorded in the field during our survey, manuring practices followed by the farmers for the tree mulberry garden was also varying. Most of the farmers (82.85\%) were using organic sources for tree mulberry cultivation and very few (17.15\%) using chemical fertilizers along with manures (Figure $1 \mathrm{E})$. As per the survey data, none of the farmers follow any appropriate dosage and schedule of fertilizer application in accordance with the existing biomass (number of shoots plus foliage).

DFL's brushed against the quantum of mulberry leaves available from the tree mulberry garden at the field

With the varied tree mulberry planting and cultivation procedures followed by the farmers, the next challenge posed is to make an appropriate decision for numbers of DFLs to be brushed against the quantum of mulberry leaves available in the tree mulberry garden. In accordance with the rough estimation of leaf availability, most of the farmers brush 100 to $200 \mathrm{dfls} /$ acre/year in the first year, 200 to $500 \mathrm{dfls} /$ acre/year in the second year and 400 to 800 dfls/acre/year from third year onwards (Figure 1j). However, irrespective of the type of the tree mulberry plantation the farmer's posses, about $77.14 \%$ of farmers brush $100 \mathrm{dfls/acre/year} \mathrm{during}$ the first year, $65.71 \%$ of farmers brush 200 to 300 DFL's during the second year and $77.41 \%$ of farmers brush 400 to $500 \mathrm{dfls} /$ acre/ year during the third year and onwards. Interestingly, few farmers even brush highest of 100 to 200 (22.85\% of farmers) dfls/acre/ year in the first year, 400 to $500 \mathrm{dfls} /$ acre/year (20\% of farmers) during the second year and 700 to $800 \mathrm{dfls} /$ acre/year $(6.45 \%$ of farmers) during the third year and onwards.
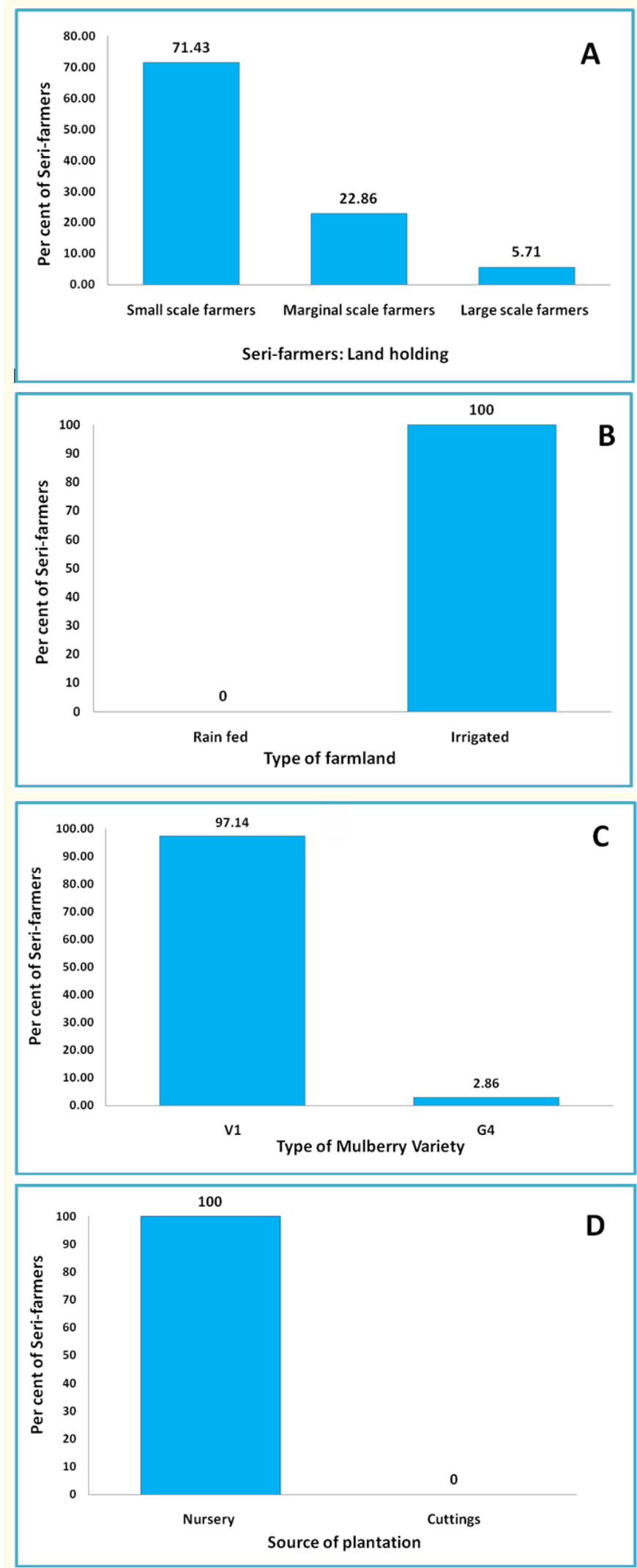

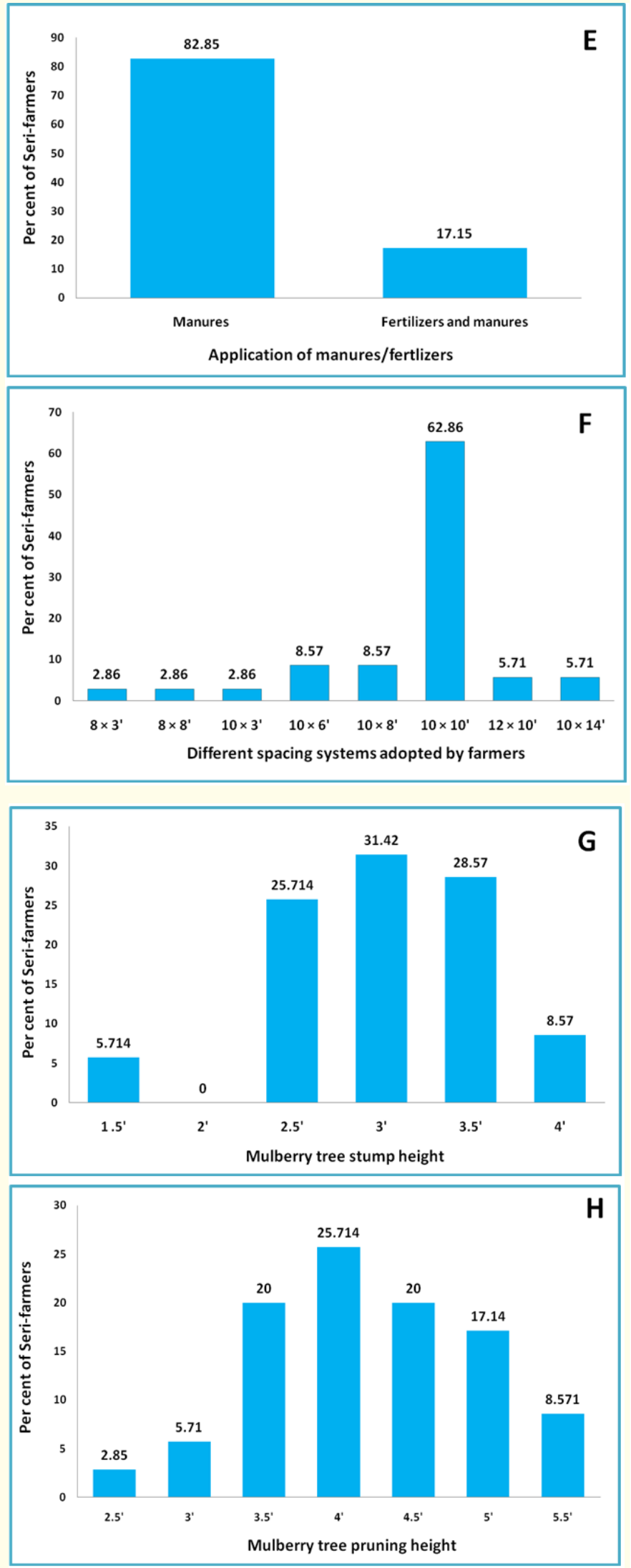


Figure 1: Different methods followed by the farmers for tree mulberry spacing, cultural operations and rearing of silkworms.

(A) Area under tree mulberry plantation; (B) Type of farmland; (C) Mulberry variety used for tree plantation; (D) Source of the plantation; (E) Percent of manures and fertilizers; (F) Spacing systems adopted by farmers; (G) Height of the stump maintained by the farmers; $(\mathrm{H})$ Pruning height from the ground level followed by the farmers; (I) Number of branches per tree maintained by the farmers; (J) Number of disease free layings brushed against the amount of leaves available from the tree mulberry garden per year.

\section{Discussion}

Tree mulberry cultivation albeit is uncommon in hilly areas like Jammu and Kashmir (India) of temperate condition to overcome frost situation, but in recent years it slowly deciphering to tropical plain areas in India. Moreover, in plain tropical areas of Karnataka, tree mulberry cultivation is in practice wherever there is a scarcity of water and manpower. Thus, more farmers are showing interest in tree mulberry plantation to overcome these two major problems following drip irrigation and mechanization. Most of the farmers in the study area use $V_{1}$ mulberry variety for tree plantation as it perform much better over other varieties in terms of productivity 

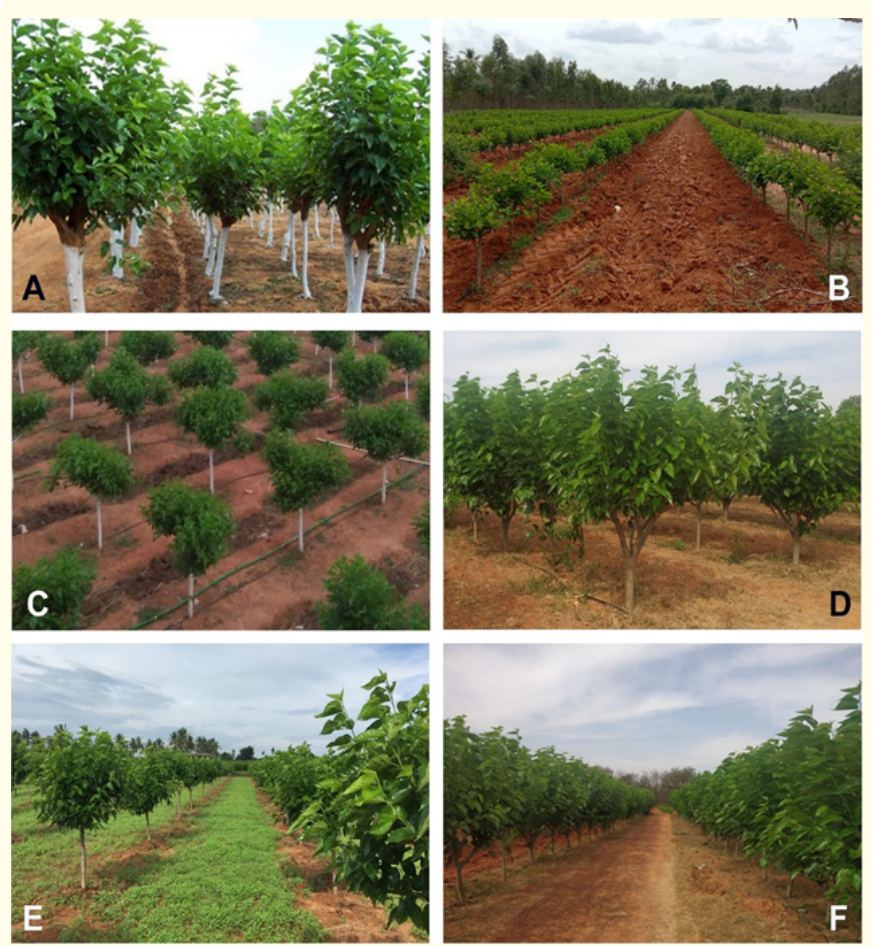

Figure 2: Different spacing adopted by the farmers for tree mulberry plantation.

(A) 5' x 5'; (B) 10' x 3'; (C) 10' x 10'; (D) 10' x 8';

(E) $14^{\prime} \times 10^{\prime} ;$ (F) 14' x 8'.

and sustenance during prolonged dry spell period. But, the major drawback noticed during the study are, wide diversity in tree mulberry geometry, cultural operations, fertilizer application and the number of disease free layings (dfls) for silkworm rearing. This diversification is due to lack of scientific basis of a recommendation that not only conflict among cultural operations but also Serifarmers, such that every farmer practice tree mulberry plantation as per their own knowledge, convenience and problems encountered over the years - as opined by the farmers.

Although Fotedar., et al. [4] has suggested 3' x 3' for the bush, 6' $x$ 6' for high bush and 9'x 9' for tree mulberry plantation for different regions, Jammu and Kashmir in particular, such defined spacing for tree plantation has not be seen followed by the farmers in Karnataka. Data from the present survey clearly explicit an arbitrary approach for tree mulberry geometry, cultural operations and number of dfls for brushing without estimating quality and quantum of leaf produced in a unit area of tree mulberry garden either for a crop or year.

Surprisingly, no standard geometry has been followed by the farmers for tree mulberry in their garden, which is on the basis of their own knowledge and convenience. As per the present data, varied spacing pattern have been followed by the farmers; about $62.86 \%$ farmers adopted the geometry of $10^{\prime} \times 10^{\prime}$ for tree mulberry even though $10^{\prime} \times 10^{\prime}$ spacing with 1093 plants/ha is recognized as non-viable due to reduction in the leaf yield up to $70 \%$ (21.08 MT/ha/year, Sudhakar., et al. [5]). As per the interaction with the farmers in the present survey, the cause for this change is due to easy mechanization, less labor requirement for intercultural operations, good exposure of leaves to sunlight and good aeration, which contributes low incidence of pest and disease, and production of good quality leaves. Contrastingly, varied stump and plant (pruning) height have also been maintained in the tree mulberry garden, which is on the basis of farmers' own knowledge and convenience but not on the line of scientific recommendation. According to our survey data, 3' and 3.5' stump height was the most followed by the farmers as it facilitates them harvesting leaves/shoots and pruning with ease, despite, a study states that 1 to $1.5^{\prime}$ stump height is convenient for cultural operations [5]. Meanwhile, as the stump height increases from 3.5' and above, it affects the pruning and shoots harvesting; if pruning height is above 5', the respondents were facing problems during shoot harvest and pruning. So, it warranted optimization of stump and pruning height in the tree mulberry garden, which could ease out farmers' constraints.

Moreover, as per the present survey data, farmers who are practicing $10^{\prime}$ x $10^{\prime}$ spacing in the tree mulberry garden allow a varied number of shoots per tree ranging from 30 to 120 . To support good growth and development of shoots with leaves both qualitatively and quantitatively superior require optimal quantum of nutrients in terms of manure or fertilizers. But, there was no such optimized manure or fertilizer dose and schedule followed in accordance with the number of shoots or biomass present in a plant. This leads to an indeterminate quantum of leaf production, which acts as a limiting factor for the farmers, as per their opinion, to decide the exact number of dfls required in order to utmost utilization of foliage for silkworm rearing. As a consequence, farmers were brushing a varied number of DFLs (100 to $800 \mathrm{dfls/acre/crop)} \mathrm{compli-}$ cating the silkworm rearing and it needs optimization in number of shoots per tree for defined quality and yield of mulberry leaves and accordingly definite number of DFLs for rearing to produce good quality cocoons.

Taken together and as per the interaction had with the farmers, tree mulberry cultivation does not found to be beneficial to some farmers, but, few farmers are performing well by employing innovative approaches as per their own knowledge and convenience. Therefore, a comprehensive and systematic investigation is underway in our tree mulberry garden with optimized geometry of $8^{\prime}$ x 5' (Figure 3A), stump height of 2.5' (Figure 3B) and pruning 
height from the ground level 3 to $3.5^{\prime}$. Accordingly, the optimized plant geometry presented here also has some supporting evidence [5], which state that 8' x 3' spacing could yield higher quantum of foliage (67.07 MT/ha/year) compared to paired row system of the bush plantation (60.14 MT/ha/year). Since, plant population is one of the significant factors to meet sustained leaf production [6] we have optimized tree mulberry geometry to $8^{\prime} \mathrm{x} 5^{\prime}$ spacing. This strategy facilitates mechanized plough, besides mixing of mulched dry leaves (Figure 3C) and/or green manure plants (sun hemp, figure 3D) grown in between mulberry tree to enrich soil health with ease. Further, as higher pruning height in the tree farm plantation compared to bush farm $(15 \mathrm{~cm})$ increase the leaf yield [4,7-9], we have optimized the stump height of $2.5^{\prime}$ and plant height of 3 to

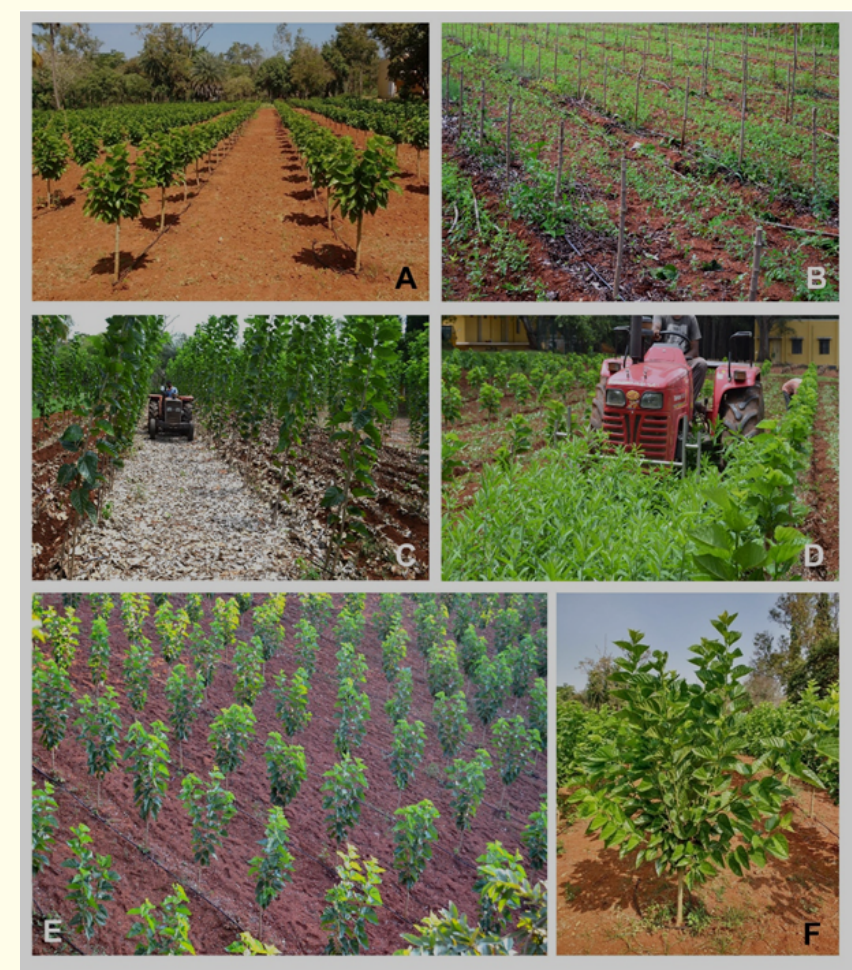

Figure 3: Optimized spacing. stump height, pruning height and number of shoots for tree mulberry plantation.

(A) The spacing provided between two rows is 8 feet and between plants is 5 feet sufficed with drip irrigation net work; (B) Uniform stump height of 2.5 feet has given to all the plants after one year of plantation; (C) The optimum space provide between two rows facilitate mechanized ploughing and mixing of mulched dry leaves to the soil with ease; (D) Mechanized incorporation of sun hemp (Green manure plants) grown in between mulberry plants to enrich soil health; (E) Well established tree mulberry garden with optimized planting geometry; (F) Well grown tree mulberry with defined number of shoots per plant to achieve assured quality and yield of mulberry leaves.
3.5' from the ground level (Figure 3B and 3F). This optimization is on the basis of difficulties encountered by the farmers (as stated above) and in-house garden personnel during shoot harvesting and pruning. Eventually, we aptly established optimized tree mulberry garden as evident in the figure 3 , but we are of the opinion that, it requires some more detailed investigation to optimize cultural operations and package of practices for long term and large scale tree mulberry cultivation with assured quality and sustained leaf production in order to undertake silkworm rearing for guaranteed cocoon production.

\section{Conclusion}

The present study uncovers substantial diversification prevailed in the tree mulberry geometry, cultural operations, fertilizer application and a number of disease free layings (DFLs) for silkworm rearing, which conflict and affecting Seri-farmers unnoticing to take-up appropriate action. Considering these limitations, we have established tree mulberry plantation with optimized plant geometry and strongly suggest the scientists and stake-holders initiate systematic research towards this end to achieve long term and large scale cultivation of tree plantation with assured quality and sustained leaf production and better mechanization. This approach not only facilitates the farmers to overcome the constraints and could brush a defined number of DFLs against mulberry leaf yield for assured and sustained cocoon production.

\section{Bibliography}

1. Dandin S B and Sengupta K. "Mulberry cultivation as high bush and small tree in hilly regions". Central Silk Board, Bangalore (1988): 1-16.

2. Dasgupta K P. "A comparative analytical study on the effect of feeding with different types of mulberry leaves obtained by different methods of cultivation on silkworm, Bombyx mori L". Indian Silk 1.4 (1961): 5-8.

3. Das P K. "Package of practices for mulberry cultivation as tree plantation for rainfed sericulture" (2010).

4. Fotedar R K., et al. "Effect of different pruning height on the mulberry yield and silkworm rearing". Indian Journal of Sericulture 24.2 (1995): 105-109.

5. Sudhakar P., et al. "Tree mulberry sustainable and economically viable sericultural farming for Southern tropical zones". International Journal of Applied and Pure Science and Agriculture 04.6 (2018): 13-23. 
6. Hasegawa K. "Study on the relations between population density and yield in the mulberry field". Bulletin Series of Experiment Station 22 (1967): 28-29.

7. Iwata E. "On the study of primary pruning to train low cutting in mulberry plants". Bulletin Series of Experiment Station (Tokyo) 27 (1977): 397-426.

8. Iwata E. "Studies on the growth of regenerated shoot after intermediate pruning and its application to cultivation of mulberry trees". Bulletin Series of Experiment Station (Tokyo) 28 (1981): 393-398.

9. Choudhury P C., et al. "Effect of spacing crown height and method of pruning on mulberry leaf yield, quality and cocoon yield". Indian Journal of Sericulture 30.1 (1991): 46-53.

Volume 5 Issue 6 June 2021

(C) All rights are reserved by Manjunatha HB. 